\title{
TSVdb: a web-tool for TCGA splicing variants analysis
}

\author{
Wenjie Sun ${ }^{1 \dagger}$, Ting Duan ${ }^{2 \dagger}$, Panmeng Ye ${ }^{3}$, Kelie Chen ${ }^{1}$, Guanling Zhang ${ }^{4}$, Maode Lai ${ }^{{ }^{*}}$ and Honghe Zhang ${ }^{1 *}$
}

\begin{abstract}
Background: Collaborative projects such as The Cancer Genome Atlas (TCGA) have generated various -omics and clinical data on cancer. Many computational tools have been developed to facilitate the study of the molecular characterization of tumors using data from the TCGA. Alternative splicing of a gene produces splicing variants, and accumulating evidence has revealed its essential role in cancer-related processes, implying the urgent need to discover tumor-specific isoforms and uncover their potential functions in tumorigenesis.

Result: We developed TSVdb, a web-based tool, to explore alternative splicing based on TCGA samples with 30 clinical variables from 33 tumors. TSVdb has an integrated and well-proportioned interface for visualization of the clinical data, gene expression, usage of exons/junctions and splicing patterns. Researchers can interpret the isoform expression variations between or across clinical subgroups and estimate the relationships between isoforms and patient prognosis. TSVdb is available at http://www.tsvdb.com, and the source code is available at https://github. com/wenjie1991/TSVdb.
\end{abstract}

Conclusion: TSVdb will inspire oncologists and accelerate isoform-level advances in cancer research.

Keywords: Splicing variant, Alternative splicing, TCGA, Cancer, Visualization tools

\section{Background}

During transcription in eukaryotes, alternative splicing (AS) of message precursor RNA generates splicing variants for a single gene, and particular exons may be included or excluded. It was estimated that approximately 92-94\% of human genes undergo AS [1]. As one of the most common mechanisms associated with gene regulation [2], AS has emerged as a vital mechanism in tumorigenesis that regulates the function of cancer-related genes [3]. Aberrant splicing patterns are closely related to tumor progression [4]. For example, misregulation of splicing caused by splicing factor Serine And Arginine Rich Splicing Factor 1 (SRSF1) can lead to the malignant transformation of normal mammary cells [5]; we also reported that Serine And Arginine Rich Splicing Factor 6 (SRSF6) promotes tumor progression by regulating AS and might be a potential therapeutic target [6]. Thus, splicing

\footnotetext{
*Correspondence: Imp@zju.edu.cn; honghezhang@zju.edu.cn

${ }^{+}$Wenjie Sun and Ting Duan contributed equally to this work.

'Department of Pathology, Key Laboratory of Disease Proteomics of Zhejiang Province, School of Medicine, Zhejiang University, 310058 Hangzhou, China

Full list of author information is available at the end of the article
}

variants could be potential biomarkers [7] and therapeutic targets in cancer studies.

The Cancer Genome Atlas (TCGA) project (http:// cancergenome.nih.gov) has incorporated a vast bulk of genomic sequences, epigenetic profiles, transcriptomes and multidimensional clinical datasets. It is an excellent source for exploring and validating genes of interest through the TCGA RNA-Seq.

Information on splicing variants can be identified from RNA Sequencing data through software such as Cufflinks, RSEM (RNA-Seq by Expectation Maximization), Kallisto and MapSplicing [8-10]. Tools such as TCGASpliceSeq [9] and ISOexpresso [11] provide users with alternative splicing patterns and isoform expression data between normal and tumor cells. However, detailed clinical information, such as the tumor grade, race and survival time, are not provided by the existing tools that investigate splicing variants.

To address these questions, we introduced TSVdb, an interactive web-portal, to perform comparative analysis on splicing variants across tumor subgroups using

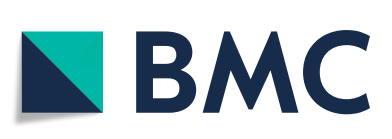
(c) The Author(s). 2018 Open Access This article is distributed under the terms of the Creative Commons Attribution 4.0
International License (http://creativecommons.org/licenses/by/4.0/), which permits unrestricted use, distribution, and reproduction in any medium, provided you give appropriate credit to the original author(s) and the source, provide a link to the Creative Commons license, and indicate if changes were made. The Creative Commons Public Domain Dedication waiver (http://creativecommons.org/publicdomain/zero/1.0/) applies to the data made available in this article, unless otherwise stated. 
TCGA RNA-Seq datasets from 33 tumors and 30 clinical variables.

TSVdb presents a well-organized visualization of exon/junction usage and splicing patterns, which enable users to readily and quickly access, analyze, and interpret splicing variants for interesting genes. Users can investigate the isoform expression between tumor subgroups and the association of splicing variant expression with overall survival.

We believe that TSVdb provides a user-friendly platform for researchers to maximize TCGA utilization and unearth more potential cancer biomarkers.

\section{Implementation \\ Data collection}

TCGA (version 20160128) level 3 data were downloaded from the TCGA FTP site Firehose. The data included the gene, isoform RSEM data, exon, and junction-normalized read count data (UNC illuminaHiSeq_RNASeqV2) and clinical data (Merged_clinical_level_1). Because the data in Firehose no longer updates, data updates will use the TCGA data in the GDC data portal in the future. The current TCGA data version was noted in the footer on the TSVdb plot page. The RNASeq data transformation was accomplished with the $\mathrm{R}$ software version 3.2.3. Genes with Entrez IDs in both the TCGA data and annotation package org.Hs.eg.db(3.2.3) were used. The annotation package TxDb.Hsapiens.UCSC.hg19.knownGene(3.2.2) was used to annotate the isoform, exon and junction data in the TCGA datasets. The annotation of the TCGA exon and junction data was performed by overlapping the locations of the exons/junctions with the isoform range. The annotation package was also used to plot the transcript isoform structure.

\section{Data manipulation and transformationn}

The related clinical information for each cancer type was selected and prepared for each tumor. Thirty clinical variables were chosen and processed (Additional file 1: Table S1). A cut-off value was used to make the numerical variables classified variables; if there were too many classes, a combination was applied to reduce the class number. The transformation methods were as follows:

(a) The number of packs smoked per year smoked, which was an integer variable. The cut-off values were set to 10 and 100 to create the following three categories: (1) "less than 10", "less than 100", and "greater than 100" packs smoked per year.

(b) Overall survival. The day_to_death and days_to_last_follow-up variables were used to generate the time and event variables for overall survival. The event was set to "death" if a patient had a defined non-zero day_to_death value; otherwise, the event variable was considered "censored". The survival time was set to the larger variable among the day_to_death and days_to_last_follow-up. The survival status was coded as 0 (live or censored) or 1 (death).

(c) Stage. The class number was reduced to 5 (I, II, III, IV, and X) for the pathology_stage, clinical_stage and masaoka_stage.

(d) Risk factors of LIHC. "Alpha-1 antitrypsin deficiency", "hemochromatosis" and "other" were combined into "others". "Alcohol consumption", "hepatitis b" and "hepatitis c" were classified by themselves due to their large proportion.

(e) Alcohol consumption per day was divided into "0" and " $>0$ ".

(f) The number of pregnancies was grouped into six classes, which defined the number of pregnancies as either "1", "2", "3", “4”, “5” or "> 5".

"Indeterminate" data were shown as "UNDEFINED". Additional file 1: Table S1 shows the phenotype statistics for the tumor types.

All the data described above were deposited into the NoSQL database MongoDB (See Additional file 2 for the database scheme).

\section{Website and Data Visualization}

The nodejs scripts were used to provide the private APIs for the web-end. There were three APIs with the following functions: (1) Autocompleting the gene symbol or alias, (2) Validating the symbol or the input Entrez ID, (3) Querying MongoDB and then returning the clinical variable list for the queried tumor, and (4) Querying MongoDB and then returning the data to draw the results. The JavaScript libraries d3.js [12] (version 3.0.6, http:// d3js.org/) were used to construct the interactive SVG graph. The d3.js-based interactive KM-plot was modified from Nick Strayer's code (http://bl.ocks.org/nstrayer/). The box plot and other distribution charts were modified from Andrew Sielen's code (https://github.com/asielen/ D3_Reusable_Charts). The SVG graph download function was adapted from A. Gordon's solution (https://github. com/agordon/d3export_demo).

$$
\begin{aligned}
& y_{i j}= \begin{cases}\min \left(\frac{x_{i j}}{e_{j} \cdot Q_{95, i}}, 1\right) & \text { if } Q_{95, i}>0.05 \\
\min \left(\frac{x_{i j}}{e_{j} \cdot 0.05}, 1\right) & \text { if } Q_{95, i} \leq 0.05\end{cases} \\
& e_{j}=\frac{\sum_{i=1}^{n} x_{i j}}{n}
\end{aligned}
$$

The exon/junction usage value $y_{i j}$ displayed in the main results (Fig. 1) was derived from the exon/junction quantification value $x_{i j}$ with a series of scalings and normalizations Eq. (1). The effects of the normalization are shown in Additional file 1: Figure S2 for a gene that 


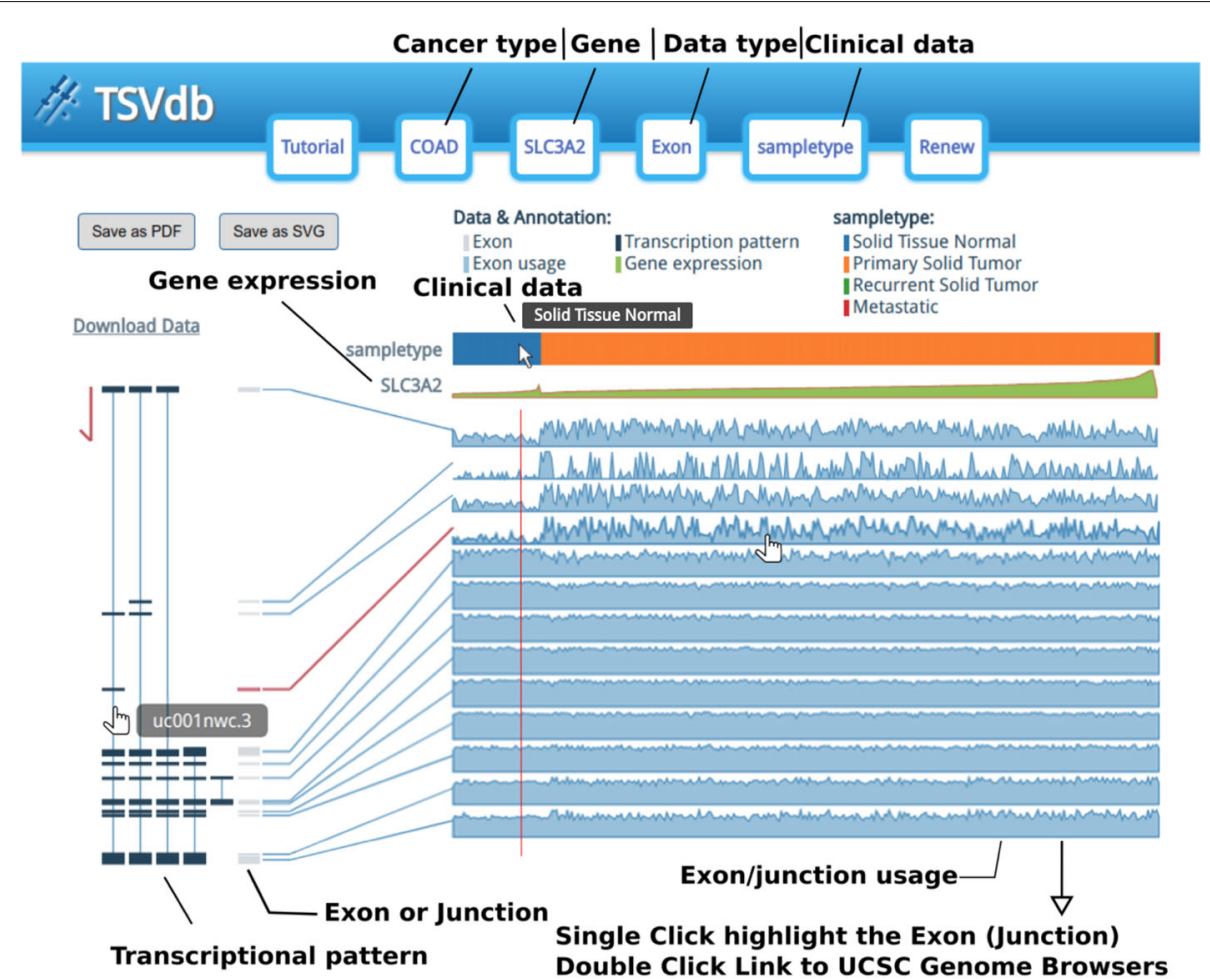

Fig. 1 An illustration of the TSVdb database. The top of the web page displays the query parameters buttons; under those buttons were the figure legends. Under the legends were the main results. The sample type, gene expression and exon/junction usage (patients are presented in columns arranged according to their gene expression from low to high in each group and the exons/junctions are arrayed in rows) are displayed on the right side and from the top down. The left side shows the gene transcriptional pattern, in which the thin lines represent the introns and boxes connected by the lines represent the exons corresponding to the rows on the right side. Hovering or clicking on the rows will highlight the corresponding exon in the transcription pattern and double clicking on the rows will open the UCSC Genome Browser in a new tab/window and showing the gene structure. Additionally, clicking on the isoform structure lead to isoform-specific expression data or a survival curve

has $n$ exon/junction values $(i=1, \ldots, n)$ in $m$ samples $(j=1, \ldots, m)$. First, following the idea of a "splicing index" [13], each sample's exon/junction quantification value was divided by the expression quantity $e_{j}$ of the gene to which the exon belongs Eq. (2). The gene expression quantity was estimated by averaging the quantification of the exons/junctions. Therefore, the gene expression effect was removed and the AS event was highlighted. Next, the d3.js linear scale was used to map the normalized exon/junction values to the graph coordination. The interval of the normalized exon/junction values (domain argument in scale function in D3) was set to $\left(0, Q_{95, i}\right)$ ( $95 \%$ quantile) to diminish the outlier's impact, which may minimize the differences in the AS events between groups. Furthermore, if $Q_{95, i}<0.05$, which indicates the exon/junction expression quantity, is relatively small when corresponding to the gene expression quantity, the upper bound of the interval would be set to 0.05 .

\section{Results}

\section{TSVdb use}

Four dialogs were initially used to input the tumor type, exon/junction and clinical data for a specific gene. After finishing the input, the main output window showed the clinical information, gene expression, exon/junction usage and isoform structure diagram (Fig. 1). As was shown, the samples were divided into two or more subgroups according to their clinical information, e.g., "Solid Tissue Normal" and "Primary Solid Tumor". The samples in each group were arrayed by their gene expression levels, which helped to distinguish the correlation between the isoform expression and gene expression. Meanwhile, the shadowed-line charts display the exon/junction usage values for each sample to facilitate the recognition of alternative splicing. Links to the UCSC Genome Browser also offer the exon/junction's loci as well as further annotation information such as the conservation of the exon sequence, single nucleotide polymorphisms, and mutations, so that researchers can gain a full-scale understanding of the exon or junction.

Furthermore, the transcriptional pattern was also displayed to reveal the splicing isoforms for a single gene and their constitutions. Notably, by double-clicking the transcriptional pattern, the expression of the isoforms in the different subgroups was shown using a box plot (Fig. 3), and the correlation of the isoforms with overall survival 
was demonstrated by a KM-plot (Fig. 4), which indicated promising use for clinical cancer researchers. The KM survival results described four different parts as follows. (1) In the bottom right, there was a way to adjust the cut-off for grouping, where the knob could be adjusted to change the cut-off. The default value was set to the middle of the isoform expression range. (2) The top right part displayed information on the groups, including the cut-off value and sample size of each group. (3) The top left showed the survival line for each entered individual after filtering them by the survival start time. The start time could be changed by adjusting the knob. (4) The bottom left was the KMplot. Moreover, users could also click on the right $y$-axis or bottom $\mathrm{x}$-axis to invoke input boxes and set the position by inputting a specific value. The formatted exon/junction quantification, transcript isoform expression, clinical variables and gene expression data for a gene in one tumor type could be downloaded into one file by clicking the download link. An illustration of the downloaded table is shown in Additional file 1: Table S2

\section{Example analysis}

The oncogene Rac Family Small GTPase 1 (RAC1) is closely associated with tumorigenesis, tumor progression and therapy resistance [14-16], and RAC1 alternative splicing is important for its regulatory role in cancers.[17]. As illustrated by RAC1 in colon cancer, RAC1 generates three splicing isoforms, and the fourth exon is skipped in normal tissues and included in tumor tissues (Fig. 2a). Consistently, it was revealed that the use of the junctions linking exons 3 to 4 and 4 to 5 were high in tumor tissues (Additional file 1: Figure S3A). Similarly, by choosing microsatellite instability (MSI) status as the phenotype, the results revealed that the fourth exon usage was

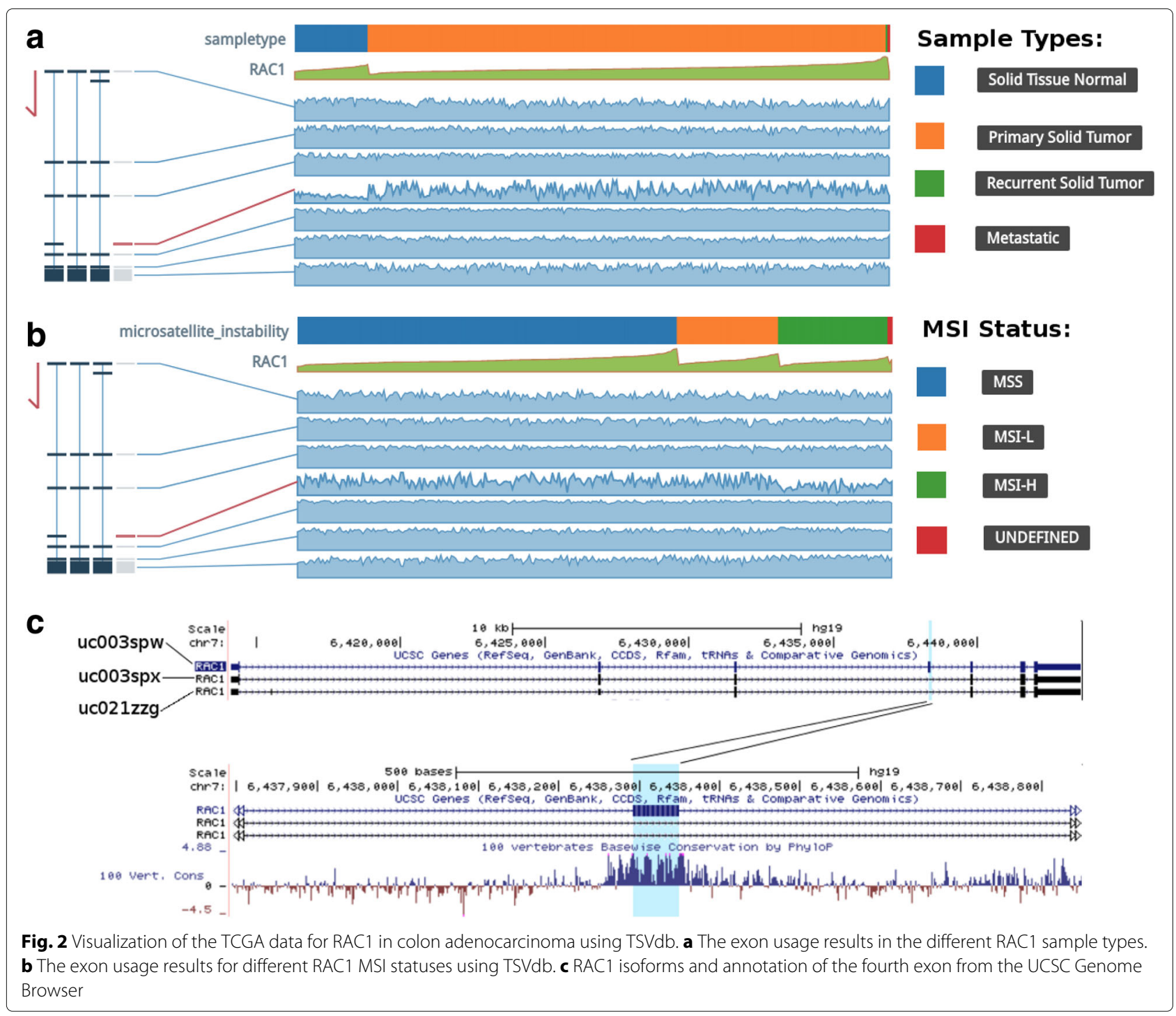


high in the microsatellite stable (MSS) and microsatellite instability-low (MSI-L) groups (Fig. 2b), suggesting its potential role in DNA damage response [18]. Moreover, annotation from the UCSC Genome Browser (Fig. 2c) showed that the DNA sequence of the fourth exon was evolutionarily conserved in vertebrates, which indicated the importance of its function in tumor biology.

The isoform expression variation for RAC1 was also shown by the box plot (Fig. 3). Primary solid tumor tissues had higher uc003spw.3 transcript expression, while there was lower uc003spx.3 transcript expression relative to normal tissues (Fig. 3a and b) Interestingly, the fourth exon was only included in isoform uc003spw.3. Furthermore, the MSI-H samples showed lower uc003spw.3 transcript expression but higher uc003spx.3 transcript expression (Fig. 3c and d). Additionally, the KM-plots showed the correlation between uc003spw. 3 and uc003spx.3 with the overall survival of colon cancer patients (Fig. 4), and the high uc003spw.3 expression was correlated with poor prognosis.

\section{Discussion}

TSVdb is a user-friendly interface for unearthing alternative splicing variations in 33 cancers. Similar to existing tools, TSVdb provides a comparison of isoform expression and alternative splicing between tumor and normal samples, which can also be achieved by ISOexpresso [11] and TCGASpliceSeq [9], respectively (Table 1).

Moreover, TSVdb presents a better and more convenient visualization for users to assess exon/junction usage, transcript isoform expression, isoform pattern graph, and clinical information in one graph. This is the first time that we have integrated comprehensive clinical data with TCGA alternative splicing analysis tools; this web-tool will help to perform comparative analysis across different tumor subgroups. The subgroups are defined by demographic data, clinical diagnosis data, treatment data and follow-up data.

By taking advantage of clinical data from TCGA, in the analysis example, the result of the gene RAC1 was shown. We found that the expression of the fourth exon was high in adenocarcinoma tumor tissue. Moreover, it was discovered that exon usage mainly increased in tumor tissues from MSS and MSI-L patients.

In the data visualization tactic aspect, Sashimi Plots are most commonly used to visualize alternative splicing [19]. Sashimi Plots use read densities to represent the amount of reads alignment in exons or junctions. There are some variations of Sashimi Plots. For example, TCGASpliceSeq uses the number to show the reads quantity and GTEx project uses the color gradient to quantify. However, Sashimi Plots cannot visualize many samples
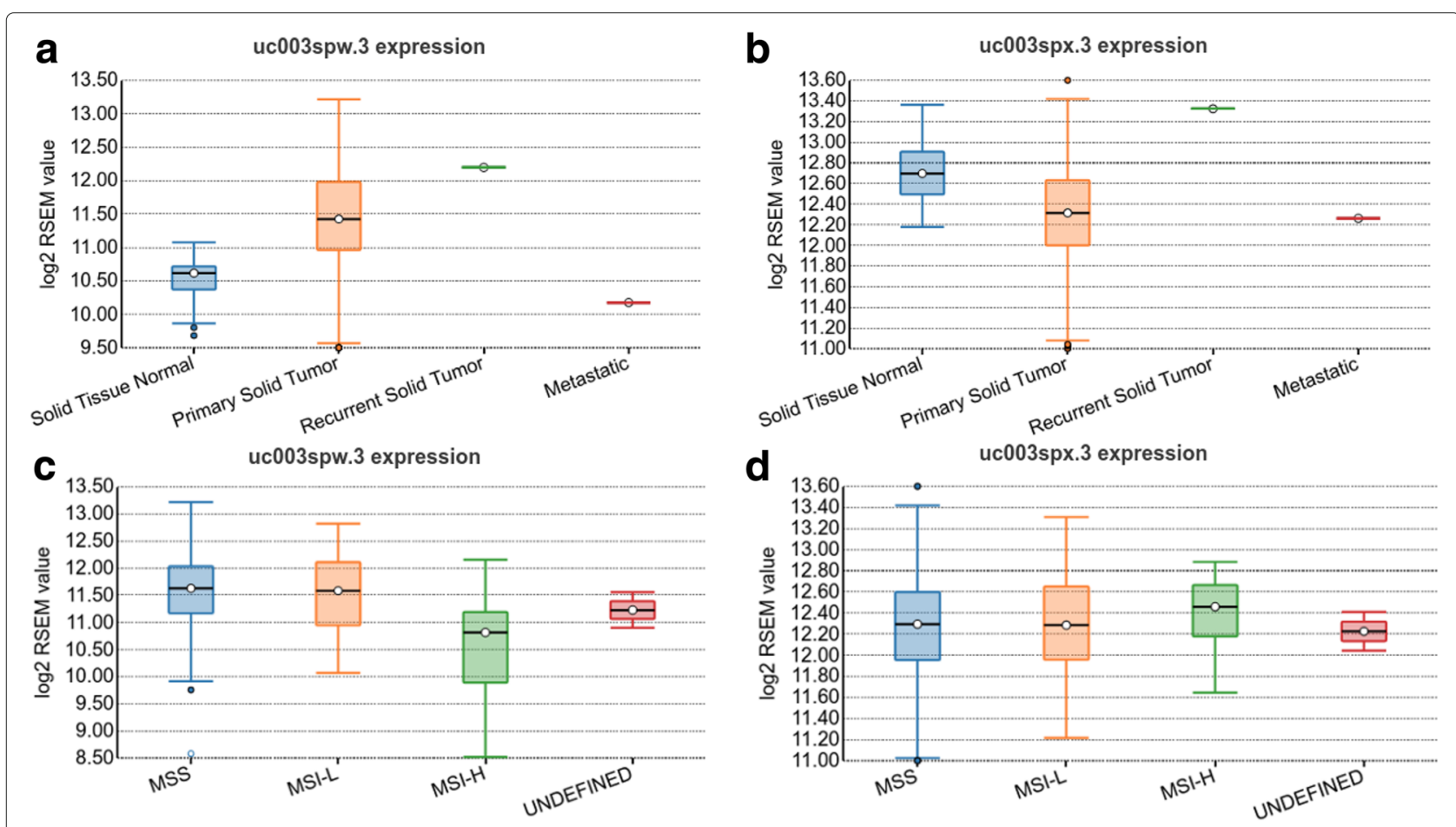

Fig. 3 Expression of RAC1 splicing isoforms in colon adenocarcinoma in TSVdb. a and $\mathbf{b}$ Differential expression of isoforms uc003spw. 3 and uc003spx.3, respectively, in tumor and normal tissues. c and $\mathbf{d}$ Differential expression of isoforms uc003spw.3 and uc003spx.3, respectively, in different MSI statuses 


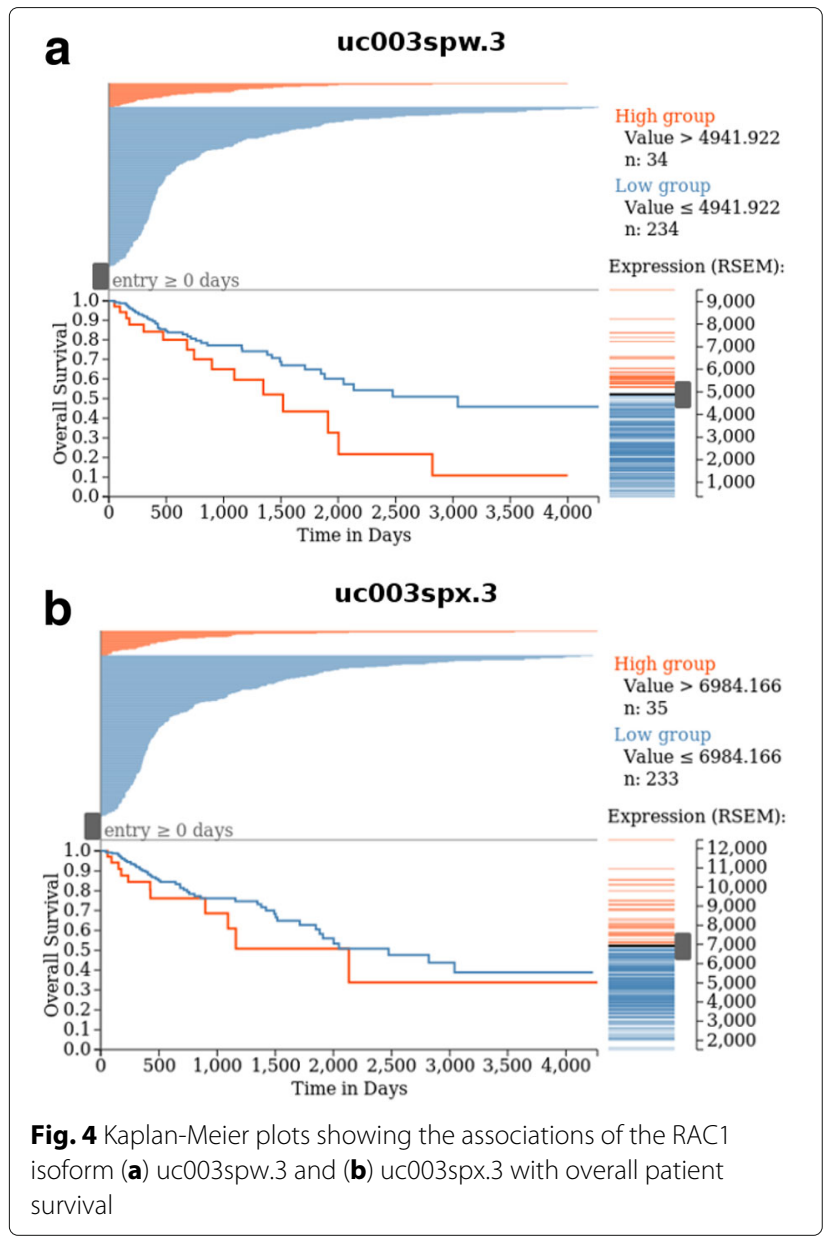

Table 1 Summary of the features of the three databases, including SpliceSeq, ISOexpresso, and TSVdb, for visualizing the TCGA splice variant data

\begin{tabular}{llll}
\hline Features & TCGA SpliceSeq & ISOexpresso & TSVdb \\
\hline $\begin{array}{l}\text { Splicing event } \\
\text { measurements }\end{array}$ & Yes & No & No \\
$\begin{array}{l}\text { Algorithm integrate } \\
\text { exon, junction reads }\end{array}$ & Yes & No & No \\
$\begin{array}{l}\text { Provide Screening } \\
\text { result }\end{array}$ & Yes & No & No \\
$\begin{array}{l}\text { Splicing pattern graph } \\
\text { Isoform pattern graph }\end{array}$ & Yes & No & No \\
$\begin{array}{l}\text { Isoform expression } \\
\begin{array}{l}\text { Exon quantification } \\
\text { Junction } \\
\text { quantification }\end{array}\end{array}$ & No & Yes & Yes \\
Clinical data & Yes & Yes & Yes \\
$\begin{array}{l}\text { Multi-omics } \\
\text { Genome-wide data }\end{array}$ & No & No & Yes \\
download & No & No & Yes \\
\hline
\end{tabular}

"ISOexpresso provided eQTL analysis

†TSVdb provided gene expression correlated AS analysis as well and do not facilitate comparisons between samples. Visualizing Alternative Splicing (Vials) (http://vials. io/vials/) resolved the problem using a complex multivariable graph [20]. In TSVdb, the data visualization strategy was inspired by MEXPRESS [21], in which samples are plotted on the $x$-axis, the genome is arranged on the $y$ axis and comparisons between subgroups are achieved by sorting the samples by phenotypes. This strategy makes it possible to display data for hundreds of samples in a single figure with genome annotations. Although, as the price, TSVdb cannot display the exon and junction reads quantity simultaneously, it can take advantage of the big sample size in TCGA datasets.

\section{Conclusion}

In summary, we provided a web-based tool for splicing variants analysis. We believe that TSVdb offers researchers a quick and straightforward visualization tool to explore alternative splicing and isoform expression of target genes in clinical subgroups within the TCGA data.

\section{Availability and requirements}

Project name: TSVdb

Project home page: http://www.tsvdb.com/

Operating system(s): Platform independent

Programming language: $R$, Nodejs and perl 6 (server side scripts)

Other requirements: Internet browser required for network visualization

License: Creative Commons Attribution 4.0 International License

(http://creativecommons.org/licenses/by/4.0/)

Any restrictions to use by non-academics: no restriction

\section{Additional files}

Additional file 1: Supplementary figures and tables. Table S1. The clinical variable distribution. Table S2. The data download format. Figure S1. The query dialogs for choosing the tumor type. Figure S2. The procedures for calculating the RAC1 exon usage in colon adenocarcinoma. Figure $\mathbf{S 3}$. The RAC1 junction usage in colon adenocarcinoma using TSVdb. Figure S4. The Kaplan-Meier plots showing the associations of the RAC1 isoform uc003spw.3, uc003spx.3 with overall patient survival. (PDF 919 kb)

Additional file 2: MongoDB database scheme. (TXT $2 \mathrm{~kb}$ )

\section{Abbreviations}

ACC: Adrenocortical carcinoma; AS: Alternative splicing; BLCA: Bladder urothelial carcinoma; BRCA: Breast invasive carcinoma; CESC: Cervical squamous cell carcinoma and endocervical adenocarcinoma; $\mathrm{CHOL}$ : Cholangiocarcinoma; COAD: Colon adenocarcinoma; DDR: DNA damage response; DLBC: Lymphoid neoplasm diffuse large B-cell lymphoma; ESCA: Esophageal carcinoma; GBM: Glioblastoma multiforme; HNSC: Head and neck squamous cell carcinoma; KICH: Kidney Chromophobe; KIRC: Kidney renal clear cell carcinoma; KIRP: Kidney renal papillary cell carcinoma; LAML: Acute myeloid leukemia; LGG: Brain lower grade glioma; LIHC: Liver hepatocellular carcinoma; LUAD: Lung adenocarcinoma; LUSC: Lung squamous cell carcinoma; MESO: Mesothelioma; MSI-H: microsatellite instability-high; MSI-L: microsatellite instability-low; MSS: microsatellite stable; OV: Ovarian serous 
cystadenocarcinoma; PAAD: Pancreatic adenocarcinoma; PCPG: Pheochromocytoma and Paraganglioma; PRAD: Prostate adenocarcinoma; RAC1: Rac family small GTPase 1; READ: Rectum adenocarcinoma; RSEM: RNA-Seq by expectation maximization; SARC: Sarcoma; SKCM: Skin cutaneous melanoma; SRSF1: Serine and arginine rich splicing factor 1; SRSF6: Serine and arginine rich splicing factor 6; STAD: Stomach adenocarcinoma; TCGA: The cancer genome atlas; TGCT: Testicular germ cell tumors; THCA: Thyroid carcinoma; THYM: Thymoma; UCEC: Uterine corpus endometrial carcinoma; UCS: Uterine carcinosarcoma; VM: Uveal melanoma

\section{Acknowledgements}

We would like to thank Ledong Wan and Riccardo Fodde who tested this website and gave us very valuable feedback. The data generated by TCGA Research Network (http://cancergenome.nih.gov/) has been used for TSVdb development.

\section{Funding}

This work is supported by grants from the National Natural Science Foundation of China (81672730 to H.Z. and 81572716 to M.L.), the 111 Project (B13026 to M.L.) and the Fundamental Research Funds for the Central Universities (172210271 to H.Z.). These funding sources had no involvement in the study design; the collection, analysis, or interpretation of data; the writing of the report; or the decision to submit the manuscript for publication.

\section{Availability of data and materials}

TSVdb is freely available for academic or commercial use at http://www.tsvdb.com/.

\section{Authors' contributions}

WS, ML, and $\mathrm{HZ}$ conceived the project. WS, PY, and KC designed and developed the TSVdb. DT and GZ participated in the designing tested the utility of the tool. WS and DT wrote and revised the manuscript. WS, TD, and GZ collected and cleared up the TCGA data. All authors have reviewd and approved the final version of this manuscript.

\section{Ethics approval and consent to participate}

Not applicable.

\section{Competing interests}

The authors declare that they have no competing interests.

\section{Publisher's Note}

Springer Nature remains neutral with regard to jurisdictional claims in published maps and institutional affiliations.

\footnotetext{
Author details

${ }^{1}$ Department of Pathology, Key Laboratory of Disease Proteomics of Zhejiang Province, School of Medicine, Zhejiang University, 310058 Hangzhou, China. ${ }^{2}$ Department of Toxicology, School of Medicine, Zhejiang University, 310058 Hangzhou, China. ${ }^{3}$ Hikvision Digital Technology, 310051 Hangzhou, China. ${ }^{4}$ Department of Integrative Biology and Physiology, University of California, Los Angeles, Los Angeles, CA, USA.
}

Received: 27 November 2017 Accepted: 10 May 2018 Published online: 29 May 2018

\section{References}

1. Wang ET, Sandberg R, Luo S, Khrebtukova I, Zhang L, Mayr C, Kingsmore SF, Schroth GP, Burge CB. Alternative isoform regulation in human tissue transcriptomes. Nature. 2008;456(7221):470-6. https://doi. org/10.1038/nature07509. Accessed 04 Sep 2017.

2. Blencowe BJ. The Relationship between Alternative Splicing and Proteomic Complexity. Trends Biochem Sci. 2017;42(6):407-8. https://doi. org/10.1016/j.tibs.2017.04.001. Accessed 20 Nov 2017.

3. Skotheim Rl, Nees M. Alternative splicing in cancer: Noise, functional, or systematic? Int J Biochem Cell Biol. 2007;39(7):1432-49. https://doi.org/ 10.1016/j.biocel.2007.02.016. Accessed 04 Sep 2017.

4. Sveen A, Kilpinen S, Ruusulehto A, Lothe RA, Skotheim RI. Aberrant RNA splicing in cancer; expression changes and driver mutations of splicing factor genes. Oncogene. 2016;35(19):2413-27. https://doi.org/10.1038/ onc.2015.318. Accessed 04 Sep 2017.
5. Anczuków O, Akerman M, Cléry A, Wu J, Shen C, Shirole NH, Raimer A Sun S, Jensen MA, Hua Y, Allain FH-T, Krainer AR. SRSF1-Regulated Alternative Splicing in Breast Cancer. Mol Cell. 2015;60(1):105-17. https://doi.org/10.1016/j.molcel.2015.09.005. Accessed 27 Oct 2017.

6. Wan L, Yu W, Shen E, Sun W, Liu Y, Kong J, Wu Y, Han F, Zhang L, Yu T, Zhou Y, Xie S, Xu E, Zhang H, Lai M. SRSF6-regulated alternative splicing that promotes tumour progression offers a therapy target for colorectal cancer. Gut. 2017;2017-314983. https://doi.org/10.1136/gutjnl-2017314983. Accessed 18 Nov 2017.

7. Omenn GS, Guan Y, Menon R. A new class of protein cancer biomarker candidates: Differentially expressed splice variants of ERBB2 (HER2/neu) and ERBB1 (EGFR) in breast cancer cell lines. J Proteome. 2014;107:103-12. https://doi.org/10.1016/j.jprot.2014.04.012. Accessed 05 Sep 2017.

8. Conesa A, Madrigal P, Tarazona S, Gomez-Cabrero D, Cervera A, McPherson A, Szcześniak MW, Gaffney DJ, Elo LL, Zhang X, Mortazavi A. A survey of best practices for RNA-seq data analysis. Genome Biol. 2016;17:13. https://doi.org/10.1186/s13059-016-0881-8. Accessed 28 Jun 2016.

9. Ryan M, Wong WC, Brown R, Akbani R, Su X, Broom B, Melott J, Weinstein J. TCGASpliceSeq a compendium of alternative mRNA splicing in cancer. Nucleic Acids Res. 2015;1288. https://doi.org/10.1093/nar/ gkv1288 00001. Accessed 19 Dec 2016.

10. Li B, Dewey CN. RSEM: accurate transcript quantification from RNA-Seq data with or without a reference genome. BMC Bioinformatics. 2011;12:323. https://doi.org/10.1186/1471-2105-12-323 Accessed 27 Oct 2017.

11. Yang IS, Son H, Kim S, Kim S. ISOexpresso: a web-based platform for isoform-level expression analysis in human cancer. BMC Genomics. 2016;17:631. https://doi.org/10.1186/s12864-016-2852-6 Accessed 26 Oct 2017.

12. Bostock M, Ogievetsky V, Heer J. D3 Data-Driven Documents. IEEE Trans Vis Comput Graph. 2011;17(12):2301-9. https://doi.org/10.1109/TVCG. 2011.185. Accessed 30 Oct 2017

13. Cuperlovic-Culf M, Belacel N, Culf AS, Ouellette RJ. Data analysis of alternative splicing microarrays. Drug Discov Today. 2006;11(21):983-90. https://doi.org/10.1016/j.drudis.2006.09.011. Accessed 08 Sep 2017.

14. Ma Q, Cavallin LE, Yan B, Zhu S, Duran EM, Wang H, Hale LP, Dong C, Cesarman E, Mesri EA, Goldschmidt-Clermont PJ. Antitumorigenesis of antioxidants in a transgenic Rac1 model of Kaposi's sarcoma. Proc Natl Acad Sci. 2009;106(21):8683-8. https://doi.org/10.1073/pnas.0812688106. Accessed 01 Nov 2017.

15. Stallings-Mann ML, Waldmann J, Zhang Y, Miller E, Gauthier ML, Visscher DW, Downey GP, Radisky ES, Fields AP, Radisky DC. Matrix metalloproteinase induction of Rac1b, a key effector of lung cancer progression. Sci Transl Med. 2012;4(142):142-95. https://doi.org/10.1126/ scitranslmed.3004062.

16. Dokmanovic $M$, Hirsch DS, Shen $Y$, Wu WJ. Rac1 contributes to trastuzumab resistance of breast cancer cells: Rac1 as a potential therapeutic target for the treatment of trastuzumab-resistant breast cancer. Mol Cancer Ther. 2009;8(6):1557-69. https://doi.org/10.1158/ 1535-7163.MCT-09-0140. Accessed 01 Nov 2017

17. Fu X-D. Both sides of the same coin: Rac1 splicing regulation by EGF signaling. Cell Res. 2017;27(4):455-6. https://doi.org/10.1038/cr.2017.19. Accessed 01 Nov 2017

18. Vilar E, Gruber SB. Microsatellite instability in colorectal cancer-the stable evidence. Nat Rev Clin Oncol. 2010;7(3):2009-237. https://doi.org/ 10.1038/nrclinonc.2009.237. Accessed 20 Nov 2017.

19. Katz Y, Wang ET, Silterra J, Schwartz S, Wong B, Thorvaldsdóttir H, Robinson JT, Mesirov JP, Airoldi EM, Burge CB. Quantitative visualization of alternative exon expression from RNA-seq data. Bioinformatics. 2015;31(14):2400-2. https://doi.org/10.1093/bioinformatics/btv034 Accessed 11 Apr 2018.

20. Strobelt $H$, Alsallakh B, Botros J, Peterson B, Borowsky M, Pfister H, Lex A Vials: Visualizing Alternative Splicing of Genes. IEEE Trans Vis Comput Graph. 2016;22(1):399-408. https://doi.org/10.1109/TVCG.2015.2467911.

21. Koch A, De Meyer T, Jeschke J, Van Criekinge W. MEXPRESS: visualizing expression, DNA methylation and clinical TCGA data. BMC Genomics. 2015;16:636. https://doi.org/10.1186/s12864-015-1847-z. Accessed 16 Nov 2016. 